\title{
Current Status and Challenges of NRF2 as a Potential Therapeutic Target for Diabetic Cardiomyopathy
}

\author{
Zhi-Dong Ge, ${ }^{1,2} \mathrm{MD}$, Qingquan Lian, ${ }^{1} \mathrm{MD}$, Xiaowen Mao, ${ }^{1} \mathrm{PhD}$ and Zhengyuan Xia, ${ }^{1,3} \mathrm{MD}$
}

\begin{abstract}
Summary
Diabetic cardiomyopathy is one of the main causes of heart failure and death in patients with diabetes mellitus. Reactive oxygen species produced excessively in diabetes mellitus cause necrosis, apoptosis, ferroptosis, inflammation, and fibrosis of the myocardium as well as impair the cardiac structure and function. It is increasingly clear that oxidative stress is a principal cause of diabetic cardiomyopathy. The transcription factor nuclear factor-erythroid 2 p45-related factor 2 (NRF2) activates the transcription of more than 200 genes in the human genome. Most of the proteins translated from these genes possess anti-oxidant, anti-inflammatory, antiapoptotic, anti-ferroptotic, and anti-fibrotic actions. There is a growing body of evidence indicating that NRF2 and its target genes are crucial in preventing high glucose-induced oxidative damage in diabetic cardiomyopathy. Recently, many natural and synthetic activators of NRF2 are shown to possess promising therapeutic effects on diabetic cardiomyopathy in animal models of diabetic cardiomyopathy. Targeting NRF2 signaling by pharmacological entities is a potential approach to ameliorating diabetic cardiomyopathy. However, the persistent high expression of NRF2 in cancer tissues also protects the growth of cancer cells. This "dark side" of NRF2 increases the challenges of using NRF2 activators to treat diabetic cardiomyopathy. In addition, some NRF2 activators were found to have off-target effects. In this review, we summarize the current status and challenges of NRF2 as a potential therapeutic target for diabetic cardiomyopathy.
\end{abstract}

Key words: Reactive oxygen species, Diabetes mellitus, Cancer

(Int Heart J 2019; 60: 512-520)

$\mathrm{D}$ iabetes mellitus affects the heart through various mechanisms including metabolic disturbance, subcellular component abnormalities, microvascular impairment, and cardiac autonomic dysfunction. ${ }^{1-3)}$ Eventually, the structure and function of the heart become impaired without coronary artery disease and hypertension, a disorder known as diabetic cardiomyopathy (DCM). ${ }^{4-7)}$ Although ischemic heart disease is the most frequent cause of heart failure and death in diabetic patients, DCM is increasingly recognized as a clinically relevant entity. ${ }^{6-9)}$ It is estimated that about $12 \%$ of the diabetic patients develop DCM. ${ }^{10)}$

The pathogenesis of DCM is predominantly related to the overgeneration of reactive oxygen species (ROS) as well as impaired antioxidant ability in diabetes mellitus. ${ }^{1,11)}$ The transcription factor nuclear factor-erythroid $2 \mathrm{p}$ 45-related factor 2 (NRF2, also called Nfe212) is a master regulator of cellular redox status and detoxification response. ${ }^{12)}$ It plays a vital role in maintaining the oxidative homeostasis by regulating multiple downstream antioxi- dants. Thus, NRF2 has been proposed to be an attractive target for DCM. Recent studies show that the activation of NRF2 by natural and synthetic substances reduces high glucose-elicited oxidative damage in cultured cardiomyocytes and prevents the development of DCM in animal models of DCM. ${ }^{13,14)}$ As a result, NRF2 has gained great attention as a promising drug target for preventing the development of DCM. On the other hand, the constitutive activation of NRF2 occurs in a variety of cancers, whose prevalence is increased in diabetic patients. The aberrant activation of NRF2 is correlated with cancer progression, chemoresistance, and radioresistance. This "dark side" of NRF2 associated with tumor risk increases the challenges of using NRF2 activators to treat DCM. In this review, we discuss oxidative stress in diabetes mellitus and the significance of NRF2 in myocardial defense against oxidative damage and evaluate several NRF2 activators that receive wide attention. We also discuss the challenges of NRF2 as a potential therapeutic target for DCM.

From the ${ }^{1}$ Department of Anesthesiology, The Second Affiliated Hospital \& Yuying Children's Hospital of Wenzhou Medical University, Wenzhou, Zhejiang, China, ${ }^{2}$ Department of Anesthesiology, Medical College of Wisconsin, Milwaukee, Wisconsin, USA and ${ }^{3}$ Department of Anesthesiology, The University of Hong Kong, Hong Kong, China.

This work was supported, in part, by a research grant 81770831 (to Dr. Zhi-Dong Ge) from the National Science Foundation of China, Beijing, The People's Republic of China.

Address for correspondence: Zhi-Dong Ge, MD, Department of Anesthesiology, Medical College of Wisconsin, 8701 Watertown Plank Road, Milwaukee, WI 53226, USA. E-mail: Wilson.ge99@gmail.com

Received for publication August 7, 2018. Revised and accepted November 2, 2018.

Released in advance online on J-STAGE April 10, 2019.

doi: 10.1536/ihj.18-476

All rights reserved by the International Heart Journal Association. 


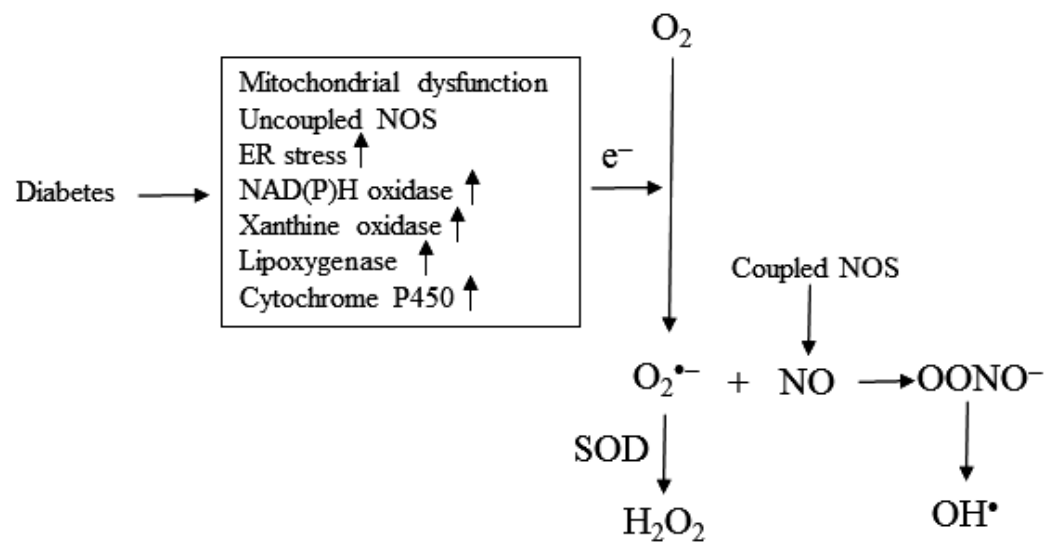

Figure 1. Production of ROS in diabetes mellitus. Diabetes mellitus results in mitochondrial dysfunction, nitric oxide synthase (NOS) uncoupling, endoplasmic reticulum (ER) stress, and up-regulates several ROS-generating enzymes, including NAD $(\mathrm{P}) \mathrm{H}$ oxidase, xanthine oxidase, and lipoxygenase, and cytochrome $\mathrm{P} 450$. These organelles or enzymes oxidize molecular oxygen $\left(\mathrm{O}_{2}\right)$ to superoxide $\left(\mathrm{O}_{2}{ }^{--}\right)$. Under normal conditions, $\mathrm{O}_{2}{ }^{--}$can be converted into the less reactive species hydrogen peroxide $\left(\mathrm{H}_{2} \mathrm{O}_{2}\right)$ by superoxide dismutase (SOD), which is further reduced into water and oxygen. In addition, superoxide can interact with coupled NOSgenerating nitric oxide (NO) to form the stronger reactive species peroxynitrite $\left(\mathrm{ONOO}^{-}\right)$, which cause tissue damage. Peroxynitrite can also be converted into hydroxyl radicals $\left(\mathrm{OH}^{*}\right)$

\section{DCM in Diabetic Patients}

According to the World Health Organization, about 422 million adults were diagnosed with diabetes mellitus worldwide in 2016. Moreover, the prevalence of diabetes mellitus has been rapidly increasing globally, especially in middle- and low-income countries. ${ }^{15,16)}$ Diabetes mellitus comprises two broad etiopathogenetic categories: type 1 (T1DM) and type 2 diabetes mellitus (T2DM). Most of the cases of T1DM result from autoimmune destruction of the $\beta$-cells of the pancreas, which leads to insulin deficiency, termed type $1 \mathrm{~A}$ diabetes mellitus. A minority of the T1DM patients have no evidence of autoimmunity but have permanent insulinopenia and are prone to ketoacidosis. This idiopathic form of diabetes mellitus is called type $1 \mathrm{~B}$ diabetes mellitus. T2DM is characterized by insulin resistance. ${ }^{17)}$ It often develops in adults and accounts for $90 \%-95 \%$ of the total diabetic population. Most patients with T2DM are obese, and the risk of developing T2DM increases with age, obesity, and lack of physical activity. ${ }^{15}$ T2DM has evolved as a rapidly increasing epidemic that parallels the increased prevalence of obesity across the globe. ${ }^{16)}$

Diabetes mellitus increases the risk of cardiovascular disease by 2 -3-fold in men and 5-fold in women compared to non-diabetic population. ${ }^{18,19)}$ The cardiovascular disease that frequently occurs in diabetic patients includes coronary heart disease, hypertension, and DCM. ${ }^{20,21)}$ DCM is characterized by left ventricular dysfunction that is independent of ischemic heart disease and hypertension. ${ }^{4,5}$ The main morphological and structural changes in the myocardium include local inflammation, myocardial fibrosis, steatosis, apoptosis, and ventricular hypertrophy. ${ }^{22)}$ It is estimated that DCM affects approximately $12 \%$ of the diabetic patients, and almost $22 \%$ of the subjects over 64 years-old. ${ }^{10)}$ It is difficult to prevent the progression of
DCM to overt heart failure. ${ }^{23,24)}$ Therefore, there is an urgent need to search new agents to treat DCM more effectively.

\section{ROS as Principal Mediators of DCM}

ROS are generated continuously as natural byproducts of the normal metabolism of oxygen. ${ }^{25}$ ) The common forms of ROS identified in the human heart include the superoxide anion $\left(\mathrm{O}_{2}{ }^{-}\right)$, the hydroxyl radical $\left(\mathrm{OH}^{*}\right)$, hydrogen peroxide $\left(\mathrm{H}_{2} \mathrm{O}_{2}\right)$, singlet oxygen, carboncentered radical, peroxynitrite $\left(\mathrm{ONOO}^{-}\right)$, nitric oxide $\left(\mathrm{NO}^{\circ}\right)$, and nitrogen dioxide radical. Under physiological conditions, the generation of ROS in the heart is low, and they are eliminated efficiently by natural antioxidant defense systems, such as the superoxide dismutase and the glutathione cycle.

ROS are excessively produced in diabetic patients and in diabetic animals through multiple pathways, including mitochondrial dysfunction, uncoupled nitric oxide synthase, endoplasmic reticulum stress, and activation and up-regulation of ROS-producing enzymes, such as NAD (P)H oxidase, cyclooxygenase, xanthine oxidase, lipoxygenase, and cytochrome P450 (Figure 1). These ROS include the highly reactive $\mathrm{O}_{2}{ }^{-}$and $\mathrm{OH}^{\cdot}{ }^{26)}$ Oxidative stress occurs when ROS production overwhelms the endogenous antioxidant ability to neutralize these highly reactive chemical compounds. The heart is highly vulnerable to oxidative stress compared with other organs and tissues due to its low basal levels of antioxidants, differences in mitogen-activated protein kinase family, and lower activity of conitase (a Krebs cycle enzyme). ${ }^{27-29}$ )

ROS overproduced in diabetes mellitus are a major causative factor for the development and progression of DCM $^{30)}$ ROS cause impairments in the structure and function of the heart by attacking, denaturing, and modifying 


\section{Cardiomyocytes}

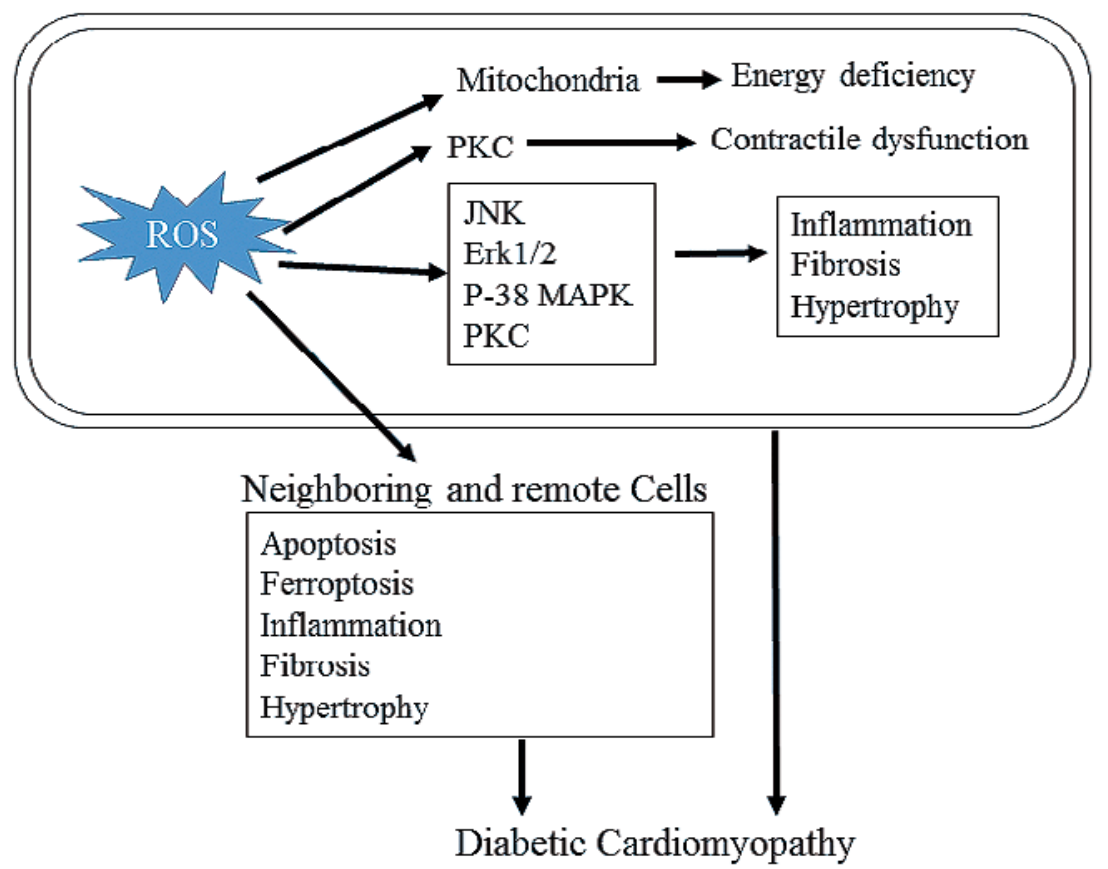

Figure 2. Pathological effects of overexpressed ROS in the diabetic heart. ROS overproduced in diabetic cardiomyocytes have autocrine and paracrine effects on the cardiomyocytes themselves and neighboring and remote cells. Within the cardiomyocytes, ROS are able to cause energy deficiency, contractile impairment, apoptosis, ferroptosis, inflammation, fibrosis, and hypertrophy through intracellular signaling pathways (p38-MAPK, PKC, and NOX) and mitochondrial mediators. ROS could also induce apoptosis, ferroptosis, inflammation, fibrosis, and hypertrophy in remote and neighboring (endothelial, vascular, and fibroblasts) cells.

functional molecules and by activating redox-sensitive transcription factors and signal transduction pathways (Figure 2). ${ }^{11)}$ These events result in energy deficiency, contractile dysfunction, necrosis, apoptosis, ferroptosis, inflammation, fibrosis, and hypertrophy of cardiomyocytes. ROS produced in cardiomyocytes have paracrine effects on neighboring and remote vascular cells and fibroblasts, leading to apoptosis, ferroptosis, inflammation, fibrosis, and hypertrophy. In vivo studies support that ROS produced excessively in diabetes mellitus cause endothelial dysfunction, microangiopathies, and atherosclerosis in blood vessels of diabetic patients. ${ }^{31)}$

\section{Critical Importance of NRF2 in Myocardial Defense Against Oxidative Damage}

NRF2 is a basic leucine zipper transcription factor with a cap ' $n$ 'collar structure. ${ }^{32)}$ It is highly expressed in normal hearts. ${ }^{33)}$ Its human sequence contains 605 amino acids, which are divided into seven domains called NRF2-ECH homology (Neh). Each of its seven domains fulfills distinct functions. The Neh1 domain contains a cap 'n'collar-basic leucine zipper motif that allows NRF2 to heterodimerize with Maf proteins and bind with DNA. The Neh2 domain contains the Kelch-like ECH-associated protein 1 (Keap1)-binding site (DLG and ETGE motifs in the N-terminal Neh2 domain), which is necessary for its cytoplasmic retention and degradation. The Neh3 domain is fundamental for NRF2 transcriptional activation through binding with chromo-ATPase/helicase DNA-binding protein 6. Neh4 and Neh5 provide interactions sites for the nuclear cofactors RAC3/AIB1/SRC-3 and CREB-binding protein, which enhances the NRF2/antioxidant response element (ARE) pathways, partially by promoting acetylation of NRF2. The Neh6 domain may contain a degron that is involved in the degradation of NRF2. The Neh7 domain interacts with retinoid $\mathrm{X}$ receptor- $\alpha$, which is responsible for NRF2/ARE signaling inhibition.

The basal activity of NRF2 as well as the magnitude of its activation in response to stress is tightly controlled. Under normal homeostatic (basal) conditions, NRF2 is kept in the cytoplasm by Keap 1 and cullin 3 (Cul3), which degrades NRF2 by ubiquitination. ${ }^{34)}$ Human KEAP1 is a $69-\mathrm{kD}$ protein that contains 27 cysteine residues. It is a substrate adaptor for Cul3, which contains E3 ubiquitin ligase activity. Cul3 ubiquitinates NRF2, while Keap1 is a substrate adaptor protein that facilitates the reaction. Once NRF2 is ubiquitinated, it is transported to the proteasome, where it is degraded and its components recycled. Under normal conditions, NRF2 has a half-life of only 20 minutes and is maintained at a low level due to proteasomal degradation. Oxidative stress or electrophilic stress disrupts critical cysteine residues in Keap1, which leads to the disruption of the Keap1-Cul3 ubiquitination system. ${ }^{35)}$ When NRF2 is not ubiquitinated, it builds up in the cytoplasm and translocates into the nucleus. ${ }^{36)}$ In the nucleus, 


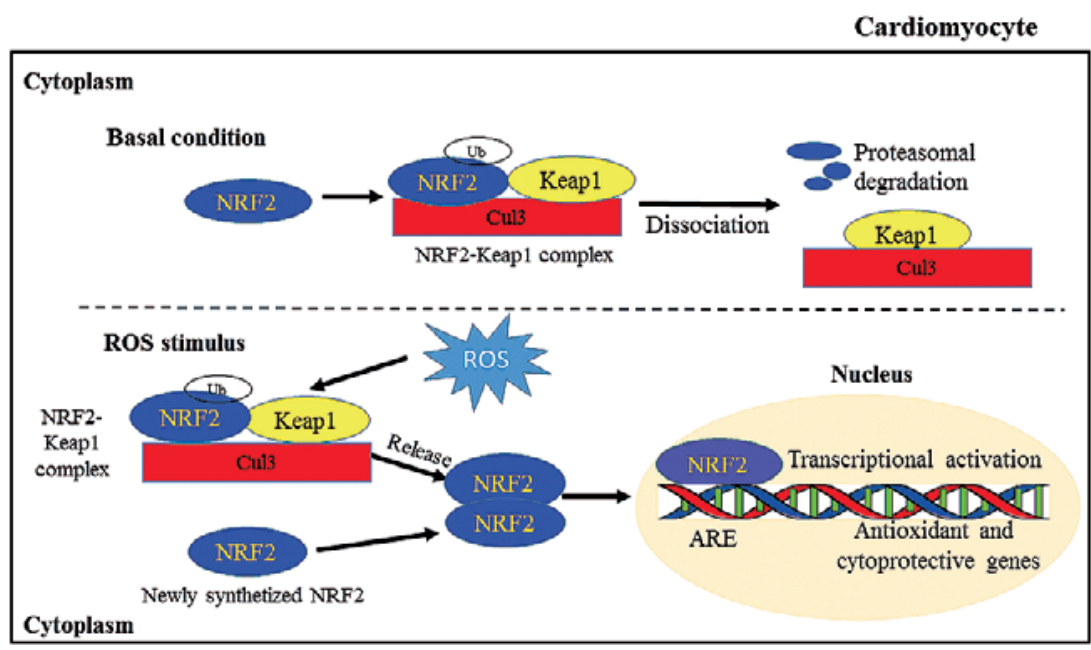

Figure 3. Schematic representation of NRF2 activation and antioxidant role in cardiomyocytes. Under basal conditions, NRF2 in the cytoplasm is constitutively bound to Keap1 and held in an inactive state. Keap1 functions to inhibit the NRF2 signaling pathway by promoting NRF2 ubiquitination through Cul3 and subsequent degradation of NRF2 by the proteasome. In responses to ROS, the NRF2-Keap1 complex is disrupted, and both the released and newly synthetized NRF2 translocates to the nucleus, where NRF2 binds to AREs in the promoter region of its target genes and promotes transcriptional activation of antioxidants and detoxifying enzymes.

NRF2 combines with one of the small Maf proteins (MAFF, MAFG, MAFK) and binds to AREs in the upstream promoter region of many antioxidative genes to stimulate transcription of antioxidative genes (Figure 2). It has been known that the activation of NRF2 results in the induction of many cytoprotective proteins, including NAD (P)H quinone oxidoreductase 1, glutamate-cysteine ligase, sulfiredoxin 1 and thioredoxin reductase 1 , heme oxygenase-1, glutathione S-transferase family, UDPglucuronosyltransferase family, and multidrug resistanceassociated proteins. ${ }^{31,37)}$

The expression of NRF2 in the heart is regulated by the gene $L A Z 3$ (BCL-6) and microRNA-21 in DCM. ${ }^{38}$ $L A Z 3$ encodes a sequence-specific transcriptional repressor which contains six zinc-finger motifs and shares aminoterminal homology with several transcription factors. ${ }^{39)}$ The small noncoding RNA microRNA-21 is an important microRNA that is regulated in all types of diabetes mellitus and heart disease. ${ }^{40-43}$ In the streptozotocin-induced cardiomyopathy of T1DM in mice, LAZ3 was downregulated and microRNA-21 was up-regulated. ${ }^{38)}$ Overexpression of $L A Z 3$ decreased the expression of microRNA21 and protected the heart against DCM, which was abrogated by the NRF2 small interfering RNA. ${ }^{38)}$ Thus, it is likely that the regulatory effect of LAZ3 on DCM is mediated by microRNA-21 and NRF2.

The NRF2/Keap1/ARE pathway is the major mechanism of myocardial defense against oxidative damage in diabetes mellitus and high glucose (Figure 3$){ }^{44)}$ This pathway regulates the expression of over 200 genes, many of which are related to reduction of oxidative stress and cell death. For example, the NRF2/Keap1/ARE signaling regulates direct antioxidant proteins, phase I and II electrophile detoxification enzymes, and free radical metabolism, inhibits inflammation, maintains glutathione homeostasis, recognizes DNA damage, as well as regulates the expression of various growth factors and transcription factors. They have been shown to attenuate oxidative stressinduced myocardial injury and prevent the progression of DCM. $^{45,46)}$

As a key master switch controlling the expression of antioxidant and protective enzymes, NRF2 modification significantly impacts the cardiac and vascular phenotype. Genetic deletion of NRF2 in mice resulted in cardiac hypertrophy, left ventricular diastolic dysfunction, and decreased responses to $\beta$-adrenergic stimulation and ouabain. ${ }^{47)}$ Left ventricular dysfunction in NRF2-null mice was associated with the down-regulation of the sarcoplasmic reticulum $\mathrm{Ca}^{2+}$-ATPase in the myocardium. ${ }^{47)}$ In addition, systemic blood pressure was decreased, and endothelial nitric oxide synthase was up-regulated in the aorta and heart of NRF2-null mice. ${ }^{47)}$

\section{Beneficial Effects of NRF2 Activators on DCM}

The above results clearly indicate that oxidative stress is a key mechanism by which diabetes mellitus induces DCM. Hence, targeting oxidative stress-related processes in DCM could be a promising strategy for preventing the development of DCM. NRF2 and its downstream signaling pathways are key in preventing high glucose-induced oxidative damage in the cardiovascular system. $^{48-51)}$ Many natural and synthetic activators of Nrf-2 have been shown to possess promising therapeutic effects on DCM in animal models of DCM. ${ }^{5,52,53)}$ Although the data regarding the clinical trials of NRF2 activators on DCM are limited, NRF2 has been considered as a promising therapeutic target for DCM (Figure 4). ${ }^{53)}$ In this section, we discuss several of the NRF2 activators that have a good potential, including sulforaphane (SFN), resveratrol 


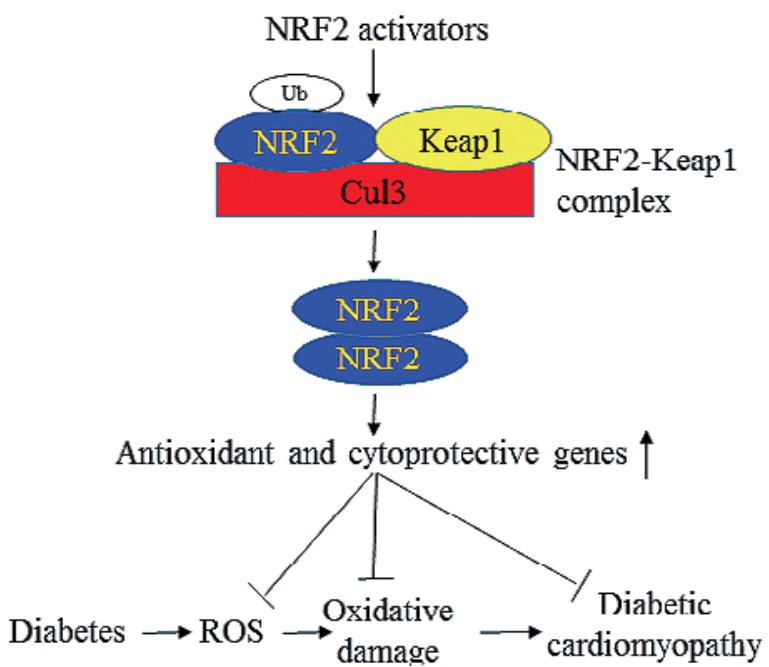

Figure 4. Schematic diagram illustrating the activation of NRF2 as a potential therapeutic target in diabetic cardiomyopathy. Diabetes mellitus elevates the production of ROS, leading to diabetic cardiomyopathy. NRF2 activators disrupt the NRF2-Keap1 complex, causing NRF2 release, or increase the expression of NRF2. In the nucleus, NRF2 promotes transcriptional activation of antioxidants and cardioprotective genes by binding to its target genes, leading to a decrease in the ROS and oxidative damage as well as to the amelioration of diabetic cardiomyopathy. Thus, NRF2 could be a therapeutic target in diabetic cardiomyopathy.

(RSV, 3,4,5-trihydroxystilbene), curcumin, dimethyl fumarate (DMF), rutin (3,3',4',5,7-pentahydroxyflavone-3rhamnoglucoside), and myricitin (Table).

SFN: SFN is a molecule within the isothiocyanate group of organosulfur compounds from cruciferous vegetables, such as broccoli, brussel sprouts, or cabbage. It has been chemically synthetized. ${ }^{54}$ As a potent NRF2 activator, SFN has been extensively studied for its effects on diabetic complications. ${ }^{50,55-57)}$ In the streptozotocin-induced mouse model of T1DM, SFN significantly up-regulated NRF2 and improved cardiac function. ${ }^{55)}$ The beneficial effects of SFN on diabetic mice are attributed to the reduction of oxidative stress-induced cardiac damage, inflammation, and fibrosis. ${ }^{55)}$ Similarly, in the mouse model of T2DM, SFN was able to activate NRF2 antioxidant signaling and prevent T2DM-induced lipotoxicity and cardiomyopathy. ${ }^{56,57)}$ Moreover, SFN reduced fasting blood glucose and glycated hemoglobin in obese patients with T2DM. ${ }^{58)}$ Translation of SFN to the clinic has been achieved by administration of SFN-containing broccoli sprout powder to patients with T2DM. ${ }^{59,60)}$

RSV: RSV exists naturally in grapes, berries, nuts, and various other plants. It is a potent NRF2 activator and inhibits oxidative stress. RSV possesses multiple pharmacologic benefits, including anti-hyperglycemic, vasodilatory, anti-fibrotic, anti-inflammatory, and anti-apoptotic effects in animal models. ${ }^{61-64)}$ Recently, a large body of preclinical evidence has shown that RSV protected the heart against DCM..$^{14,62,65-71)}$ There are some clinical data regarding the favorable effects of RSV on DCM. ${ }^{72,73)}$ The high safety profile of RSV coupled with its multidirectional effects has made it an attractive candidate to use in the clinical trial of DCM.

Curcumin: Curcumin is a natural compound isolated from turmeric. It has been used for the treatment of obesity, metabolic syndrome, and prediabetes. ${ }^{74-76)}$ In the streptozotocin-induced T1DM in rats, curcumin attenuated oxidative-induced cardiac damage, myocardial hypertrophy, and apoptosis, thereby ameliorating the function of the left ventricle..$^{77-79)}$ Curcumin activated the NRF2 system, repressed inflammation, and decreased the levels of fasting blood glucose and glycated hemoglobin in T2DM patients. $^{74-76)}$ The protective effect of curcumin on DCM is associated with increased heme oxygenase-1, catalase, superoxide dismutase, and glutathione and decreased expression of the $\mathrm{NAD}(\mathrm{P}) \mathrm{H}$ oxidase subunits $\mathrm{p} 22$ phox, 447 phox, p67 phox, and gp91 phox. ${ }^{77,78,80)}$

DMF: DMF is an electrophilic compound and is marketed under the name Tecfidera ${ }^{\circledR}$. It stimulates the NRF2 transcriptional pathway to induce anti-oxidant and antiinflammatory phase II enzymes to prevent chronic neurodegeneration. ${ }^{81)}$ DMF is currently used as the first line of treatment in patients with relapsing multiple sclerosis. The monoester form of DMF, monomethyl fumarate, is its active metabolite. Recently, DMF was shown to improve cardiac function in a mouse model of T1DM. ${ }^{13)}$ This favorable effect of DMF is attributed to the activation of the NRF2 signaling pathway, which results in the attenuation of oxidative stress, inflammation, fibrosis, and endothelial dysfunction. ${ }^{13)}$ New formulations of DMF are being tested to improve the drug bioavailability and efficacy. ${ }^{82}$ These new formulations of DMF may have potential to use in the clinical trial of DCM. ${ }^{83}$

Rutin: Rutin is a flavonol. It can be extracted from many nature sources, including buckwheat, oranges, grapes, lemons, limes, peaches, and berries. Rutin is a phytochemical with multiple pharmacological activities, including anti-diabetic, anti-oxidative, and free radicalscavenging bioactivities. ${ }^{84)}$ Many studies have shown that rutin alleviated DCM and improved cardiac function in animal models of both T1DM and T2DM.$^{85-90)}$ Mechanistically, the protective effects of rutin on DCM are associated with the reduction of oxidative stress, apoptosis, and inflammation. ${ }^{85}$ )

Myricitin: Myricetin is a widely distributed flavonol compound found in many plants, including tea, berries, fruits, vegetables, wines, and medicinal herbs. ${ }^{91)}$ This phenolic compound exhibits a wide range of activities including strong anti-oxidant, anticancer, antidiabetic, antiinflammatory, antihypertensive, and immunomodulatory activities. ${ }^{91)}$ Emerging studies indicate that myricetin alleviated cardiac hypertrophy, apoptosis, and interstitial fibrosis, leading to attenuation of DCM in the animal model of T1DM. ${ }^{92)}$ The protective effects of myricetin on $\mathrm{DCM}$ are associated with the inhibition of $\mathrm{I} \kappa \mathrm{B} \alpha / \mathrm{NF} \mathrm{KB}$ and enhancement of NRF2/heme oxygenase- 1 and mitochondrial density. ${ }^{92,93)}$

\section{Concerns about Facilitating Tumor Growth of NRF2 Activators}

NRF2 is aberrantly accumulated in many types of cancers, including lung, breast, ovarian, head and neck, 
Table. Beneficial Effects of NRF2 Activators on Diabetic Cardiomyopathy in Experimental Animals

\begin{tabular}{|c|c|c|c|c|}
\hline Compounds & Animal models & Effects & Main mechanisms & References \\
\hline Sulforaphane & T1MD mice & $\begin{array}{c}\text { Ameliorates DCM } \\
\text { Prevents diabetes-induced cardiac oxidative damage } \\
\text { Attenuates inflammation and fibrosis }\end{array}$ & Up-regulates NRF2 & 50 \\
\hline Sulforaphane & T2MD mice & $\begin{array}{c}\text { Ameliorates DCM } \\
\text { Represses oxidative stress } \\
\text { Attenuates inflammation and fibrosis }\end{array}$ & $\begin{array}{l}\text { Up-regulates NRF2 } \\
\text { Enhances NRF2 activity }\end{array}$ & 51,52 \\
\hline Resveratrol & T1MD rats & $\begin{array}{c}\text { Ameliorates DCM } \\
\text { Represses oxidative stress } \\
\text { Reduces apoptosis }\end{array}$ & Activates NRF2 & 64 \\
\hline Resveratrol & T1MD mice & $\begin{array}{l}\text { Ameliorates DCM } \\
\text { Represses oxidative stress } \\
\text { Attenuates fibrosis }\end{array}$ & Increases NRF2 expression & 8 \\
\hline Curcumin & T1MD rats & $\begin{array}{c}\text { Ameliorates DCM } \\
\text { Represses oxidative stress } \\
\text { Attenuates apoptosis, inflammation, and fibrosis }\end{array}$ & $\begin{array}{l}\text { Increases Akt and GSK-3 } \beta \text { phosphorylation, } \\
\text { inhibits protein kinase C-MAPK-pathway }\end{array}$ & 72,73 \\
\hline $\begin{array}{l}\text { Dimethyl } \\
\text { fumarate }\end{array}$ & T1MD mice & $\begin{array}{c}\text { Ameliorates DCM } \\
\text { Represses oxidative stress } \\
\text { Attenuates inflammation and fibrosis }\end{array}$ & Activates NRF2 & 7 \\
\hline Rutin & T1MD rats & $\begin{array}{c}\text { Ameliorates DCM } \\
\text { Represses oxidative stress } \\
\text { Attenuates inflammation and fibrosis }\end{array}$ & & 80,83 \\
\hline Rutin & T2MD mice & $\begin{array}{c}\text { Ameliorates DCM } \\
\text { Represses oxidative stress } \\
\text { Attenuates fibrosis } \\
\text { Promotes angiogenesis }\end{array}$ & & 84 \\
\hline
\end{tabular}

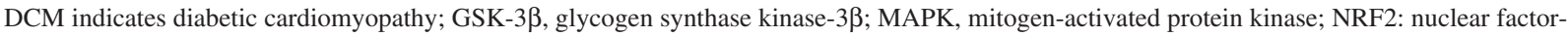
erythroid 2 p45-related factor 2; T1DM, type 1 diabetes mellitus; and T2DM, type 2 diabetes mellitus.

and endometrial cancers in humans. ${ }^{94,95)}$ Persistent high expression of NRF2 in cancer tissues protected cancer cells from chemotherapeutic agents and facilitated cancer progression. ${ }^{96)}$ Overexpression of NRF2 is associated with a poor prognosis in cancer patients. ${ }^{97)}$ Moreover, NRF2-null mice are more prone to develop cancer in response to chemical and physical stimuli (nitrosamine, ultraviolet light, aflatoxin). ${ }^{98}$ These facts suggest that NRF2 has a "dark side" related to its oncogenic activity when constitutively and highly overexpressed. Although a number of NRF2 activators have been evaluated for efficacy in humans (e.g., dithiolethiones, isothiocyanates, and triterpenoids), there is no evidence indicating direct genotoxicity and enhancing tumor growth of these agents to date.

\section{Challenges of NRF2 Activators to Treat DCM}

Diabetes mellitus and cancers are common diseases with a tremendous impact on health worldwide. Epidemiologic evidence suggests that people with diabetes are at significantly higher risk for many forms of cancers. ${ }^{99,100)}$ Oxidative stress plays a fundamental role in the pathogenesis of both DCM and cancers. The activation of NRF2 signaling pathway may benefit cardiovascular and tumor tissues in diabetes with tumors. Although there is no evidence that NRF2 activators may increase cancer occurrence, chronic utilization of NRF2 activators in cancer patients may enhance tumor metastasis and worsen prognosis. ${ }^{101)}$ Future research needs to examine the effects of new NRF2 activators on both diabetic hearts and tumor tissues simultaneously in animal models of DCM with a variety of cancers.
Another challenge of NRF2 activators to treat DCM is cytotoxicity due to off-target effects. There is mounting evidence that NRF2 can cross-talk with many intracellular signaling pathways that are important for cell survival. ${ }^{102)}$ Many NRF2 activators, such as SFN, dithiolethiones, and 1 [ 2-Cyano-3,12-dioxooleana-1,9 ( 11 ) -dien-28-oyl] imidazole, react with cysteines, leading to the modulation of multiple intracellular signaling pathways. ${ }^{103)}$ Bardoxolone methyl was withdrawn from a phase III clinical trial in end-stage renal disease patients with T2DM resulting from unspecified serious adverse effects and mortality in the treated patients. ${ }^{104)}$ The adverse events associated with bardoxolone are due to off-target events rather than the activation of the NRF2-Keap1 signaling pathway. The identification of the cocrystal structure of Keap1 in complex with the Neh2 domain of NRF2 will provide opportunities to design molecules that specifically and selectively interfere with the binding of Keap1 and NRF2.

Ferroptosis is a recently discovered form of cell death, which has immense implications for both health and disease. ${ }^{105)}$ It is a non-apoptotic regulated cell death caused by uncontrolled iron-dependent lipid peroxidation. Ferroptosis is distinct in its morphological, biochemical, and genetic profile from other cell death mechanisms. ${ }^{106)}$ Almost all genes thus far implicated in ferroptosis are transcriptionally regulated by NRF2, including the genes for glutathione regulation, NADPH regeneration, which is critical for Gpx4 activity, lipid peroxidation, and iron regulation. ${ }^{107,108)}$ Although NRF2 activators likely have an integral and pervasive impact on the manifestation of ferroptosis, ${ }^{107,108)}$ little is known regarding the role and importance of altered ferroptosis by NRF2 activators in the 
pathogenesis and development of DCM.

\section{Conclusion}

In summary, oxidative stress plays a vital role in the pathogenesis of DCM. As the center of the body antioxidant system, the NRF2/Keap1/ARE pathway is of great value in the treatment of DCM. There is a growing body of convincing experimental evidence demonstrating that pharmacological activation of NRF2 is beneficial in counteracting many of the pathological processes that occur in DCM. Although there are some challenges of the utilization of NRF2 activators to improve DCM, targeting NRF2 signaling by pharmacological entities may provide a therapeutic option to ameliorate DCM.

\section{Disclosure}

Conflicts of interest: The authors declare no conflicts of interest.

\section{References}

1. Marwick TH, Ritchie R, Shaw JE, Kaye D. Implications of underlying mechanisms for the recognition and management of diabetic cardiomyopathy. J Am Coll Cardiol 2018; 71: 339-51.

2. Pant T, Dhanasekaran A, Fang J, et al. Current status and strategies of long noncoding RNA research for diabetic cardiomyopathy. BMC Cardiovasc Disord 2018; 18: 197.

3. Ohno S, Kohjitani A, Miyata M, et al. Recovery of endothelial function after minor-to-moderate surgery is impaired by diabetes mellitus, obesity, hyperuricemia and sevofluranebased anesthesia. Int Heart J 2018; 59: 559-65.

4. Baumgardt SL, Paterson M, Leucker TM, et al. Chronic coadministration of sepiapterin and L-citrulline ameliorates diabetic cardiomyopathy and myocardial ischemia/reperfusion injury in obese type 2 diabetic mice. Circ Heart Fail 2016; 9: $\mathrm{e} 002424$

5. Wu HE, Baumgardt SL, Fang J, et al. Cardiomyocyte GTP cyclohydrolase 1 protects the heart against diabetic cardiomyopathy. Sci Rep 2016; 6: 27925.

6. Funabashi N, Takaoka H, Ozawa K, Kobayashi Y. Endocardial fibrotic lesions have a greater effect on peak longitudinal strain than epicardial fibrotic lesions in hypertrophic cardiomyopathy patients. Int Heart J 2018; 59: 347-53.

7. Takaoka H, Funabashi N, Ozawa K, et al. Improved diagnosis of detection of late enhancement in left ventricular myocardium using 2nd generation 320-slice ct reconstructed with first in non-ischemic cardiomyopathy. Int Heart J 2018; 59 542-9.

8. Mizamtsidi M, Paschou SA, Grapsa J, Vryonidou A. Diabetic cardiomyopathy: A clinical entity or a cluster of molecular heart changes? Eur J Clin Invest 2016; 46: 947-53.

9. Jia G, Hill MA, Sowers JR. Diabetic cardiomyopathy: An update of mechanisms contributing to this clinical entity. Circ Res 2018; 122: 624-38.

10. Trachanas K, Sideris S, Aggeli C, et al. Diabetic cardiomyopathy: From pathophysiology to treatment. Hellenic J Cardiol 2014; 55: 411-21

11. Shah MS, Brownlee M. Molecular and cellular mechanisms of cardiovascular disorders in diabetes. Circ Res 2016; 118: 1808-29.

12. Chen QM, Maltagliati AJ. Nrf2 at the heart of oxidative stress and cardiac protection. Physiol Genomics 2018; 50: 77-97.
13. Hu X, Rajesh M, Zhang J, et al. Protection by dimethyl fumarate against diabetic cardiomyopathy in type 1 diabetic mice likely via activation of nuclear factor erythroid-2 related factor 2. Toxicol Lett 2018; 287: 131-41.

14. Wang G, Song X, Zhao L, Li Z, Liu B. Resveratrol prevents diabetic cardiomyopathy by increasing Nrf2 expression and transcriptional activity. Biomed Res Int 2018; 2018: 2150218.

15. Hu FB. Globalization of diabetes: The role of diet, lifestyle, and genes. Diabetes Care 2011; 34: 1249-57.

16. Xu Y, Wang L, He J, et al. Prevalence and control of diabetes in chinese adults. JAMA 2013; 310: 948-59.

17. Kahn SE, Cooper ME, Del Prato S. Pathophysiology and treatment of type 2 diabetes: Perspectives on the past, present, and future. Lancet 2014; 383: 1068-83.

18. Qazi MU, Malik S. Diabetes and cardiovascular disease: Original insights from the framingham heart study. Glob Heart 2013; 8: 43-8.

19. Kengne AP, Turnbull F, MacMahon S. The framingham study, diabetes mellitus and cardiovascular disease: Turning back the clock. Prog Cardiovasc Dis 2010; 53: 45-51.

20. Benjamin EJ, Blaha MJ, Chiuve SE, et al. Heart disease and stroke statistics-2017 update: A report from the american heart association. Circulation 2017; 135: e146-603.

21. Naito R, Miyauchi K. Coronary artery disease and type 2 diabetes mellitus. Int Heart J 2017; 58: 475-80.

22. Fuentes-Antras J, Picatoste B, Gomez-Hernandez A, Egido J, Tunon J, Lorenzo O. Updating experimental models of diabetic cardiomyopathy. J Diabetes Res 2015; 2015: 656795.

23. Marcinkiewicz A, Ostrowski S, Drzewoski J. Can the onset of heart failure be delayed by treating diabetic cardiomyopathy? Diabetol Metab Syndr 2017; 9: 21.

24. Paulus WJ, Dal Canto E. Distinct myocardial targets for diabetes therapy in heart failure with preserved or reduced ejection fraction. JACC Heart Fail 2018; 6: 1-7.

25. Zhou S, Sun W, Zhang Z, Zheng Y. The role of Nrf2mediated pathway in cardiac remodeling and heart failure. Oxid Med Cell Longev 2014; 2014: 260429.

26. Wright E Jr, Scism-Bacon JL, Glass LC. Oxidative stress in type 2 diabetes: The role of fasting and postprandial glycaemia. Int J Clin Pract 2006; 60: 308-14

27. Chen Y, Saari JT, Kang YJ. Weak antioxidant defenses make the heart a target for damage in copper-deficient rats. Free Radic Biol Med 1994; 17: 529-36.

28. Zhang X, Azhar G, Nagano K, Wei JY. Differential vulnerability to oxidative stress in rat cardiac myocytes versus fibroblasts. J Am Coll Cardiol 2001; 38: 2055-62.

29. Sheeran FL, Pepe S. Posttranslational modifications and dysfunction of mitochondrial enzymes in human heart failure. Am J Physiol Endocrinol Metab 2016; 311: E449-60.

30. Thandavarayan RA, Giridharan VV, Watanabe K, Konishi T. Diabetic cardiomyopathy and oxidative stress: Role of antioxidants. Cardiovasc Hematol Agents Med Chem 2011; 9: 225-30.

31. Sharma A, Rizky L, Stefanovic N, et al. The nuclear factor (erythroid-derived 2)-like 2 (Nrf2) activator dh404 protects against diabetes-induced endothelial dysfunction. Cardiovasc Diabetol 2017; 16: 33

32. Moi P, Chan K, Asunis I, Cao A, Kan YW. Isolation of nf-e2related factor 2 (Nrf2), a nf-e2-like basic leucine zipper transcriptional activator that binds to the tandem nf-e2/ap1 repeat of the beta-globin locus control region. Proc Natl Acad Sci U S A 1994; 91: 9926-30

33. Li J, Ichikawa T, Janicki JS, Cui T. Targeting the Nrf2 pathway against cardiovascular disease. Expert Opin Ther Targets 2009; 13: 785-94.

34. Iso T, Suzuki T, Baird L, Yamamoto M. Absolute amounts and status of the Nrf2-keap1-cul3 complex within cells. Mol Cell Biol 2016; 36: 3100-12.

35. Mann GE, Rowlands DJ, Li FY, de Winter P, Siow RC. Activation of endothelial nitric oxide synthase by dietary isofla- 
vones: Role of no in Nrf2-mediated antioxidant gene expression. Cardiovasc Res 2007; 75: 261-74.

36. Hayes JD, Dinkova-Kostova AT. The Nrf2 regulatory network provides an interface between redox and intermediary metabolism. Trends Biochem Sci 2014; 39: 199-218.

37. Jung KA, Kwak MK. The Nrf2 system as a potential target for the development of indirect antioxidants. Molecules 2010; 15: 7266-91.

38. Gao L, Liu Y, Guo S, Xiao L, Wu L, Wang Z, Liang C, Yao $\mathrm{R}$, Zhang Y. LAZ3 protects cardiac remodeling in diabetic cardiomyopathy via regulating Mir-21/PPARa signaling. Biochim Biophys Acta Mol Basis Dis 2018; 1864: 3322-38.

39. Kerckaert JP, Deweindt C, Tilly H, Quief S, Lecocq G, Bastard C. Laz3, a novel zinc-finger encoding gene, is disrupted by recurring chromosome $3 \mathrm{q} 27$ translocations in human lymphomas. Nat Genet 1993; 5: 66-70.

40. Qiao S, Olson JM, Paterson M, et al. MicroRNA-21 mediates isoflurane-induced cardioprotection against ischemiareperfusion injury via Akt/nitric oxide synthase/mitochondrial permeability transition pore pathway. Anesthesiology 2015; 123: 786-98.

41. Olson JM, Yan Y, Bai X, et al. Up-regulation of microRNA21 mediates isoflurane-induced protection of cardiomyocytes. Anesthesiology 2015; 122: 795-805.

42. Sekar D, Venugopal B, Sekar P, Ramalingam K. Role of microRNA 21 in diabetes and associated/related diseases. Gene 2016; 582: 14-8.

43. Ge ZD, Li Y, Qiao S, et al. Failure of isoflurane cardiac preconditioning in obese type 2 diabetic mice involves aberrant regulation of microRNA-21, endothelial nitric-oxide synthase, and mitochondrial complex i. Anesthesiology 2018; 128: 11729.

44. David JA, Rifkin WJ, Rabbani PS, Ceradini DJ. The Nrf2/ Keap1/are pathway and oxidative stress as a therapeutic target in type II diabetes mellitus. J Diabetes Res 2017; 2017: 4826724.

45. Zhu H, Itoh K, Yamamoto M, Zweier JL, Li Y. Role of Nrf2 signaling in regulation of antioxidants and phase 2 enzymes in cardiac fibroblasts: Protection against reactive oxygen and nitrogen species-induced cell injury. FEBS Lett 2005; 579: 3029-36.

46. Dludla PV, Muller CJ, Joubert E, et al. Aspalathin protects the heart against hyperglycemia-induced oxidative damage by upregulating Nrf2 expression. Molecules 2017; 22: E129.

47. Erkens R, Kramer CM, Luckstadt W, et al. Left ventricular diastolic dysfunction in Nrf2 knock out mice is associated with cardiac hypertrophy, decreased expression of SERCA2a, and preserved endothelial function. Free Radic Biol Med 2015; 89: 906-17.

48. He X, Kan H, Cai L, Ma Q. Nrf2 is critical in defense against high glucose-induced oxidative damage in cardiomyocytes. J Mol Cell Cardiol 2009; 46: 47-58.

49. Xue M, Qian Q, Adaikalakoteswari A, Rabbani N, BabaeiJadidi R, Thornalley PJ. Activation of nf-e2-related factor-2 reverses biochemical dysfunction of endothelial cells induced by hyperglycemia linked to vascular disease. Diabetes 2008; 57: 2809-17.

50. Gu J, Cheng Y, Wu H, et al. Metallothionein is downstream of Nrf2 and partially mediates sulforaphane prevention of diabetic cardiomyopathy. Diabetes 2017; 66: 529-42.

51. Robson R, Kundur AR, Singh I. Oxidative stress biomarkers in type 2 diabetes mellitus for assessment of cardiovascular disease risk. Diabetes Metab Syndr 2018; 12: 455-62.

52. Zhou S, Jin J, Bai T, Sachleben LR Jr, Cai L, Zheng Y. Potential drugs which activate nuclear factor e2-related factor 2 signaling to prevent diabetic cardiovascular complications: A focus on fumaric acid esters. Life Sci 2015; 134: 56-62.

53. Yan B, Ren J, Zhang Q, et al. Antioxidative effects of natural products on diabetic cardiomyopathy. J Diabetes Res 2017; 2017: 2070178.
54. Kim T, Kim YJ, Han IH, et al. The synthesis of sulforaphane analogues and their protection effect against cisplatin induced cytotoxicity in kidney cells. Bioorg Med Chem Lett 2015; 25: 62-6.

55. Bai Y, Cui W, Xin Y, et al. Prevention by sulforaphane of diabetic cardiomyopathy is associated with up-regulation of Nrf2 expression and transcription activation. J Mol Cell Cardiol 2013; 57: 82-95.

56. Zhang Z, Wang S, Zhou S, et al. Sulforaphane prevents the development of cardiomyopathy in type 2 diabetic mice probably by reversing oxidative stress-induced inhibition of LKB 1/AMPK pathway. J Mol Cell Cardiol 2014; 77: 42-52.

57. Xu Z, Wang S, Ji H, et al. Broccoli sprout extract prevents diabetic cardiomyopathy via Nrf2 activation in db/db T2DM mice. Sci Rep 2016; 6: 30252.

58. Axelsson AS, Tubbs E, Mecham B, et al. Sulforaphane reduces hepatic glucose production and improves glucose control in patients with type 2 diabetes. Sci Transl Med 2017; 9: eaah4477.

59. Bahadoran Z, Mirmiran P, Hosseinpanah F, Rajab A, Asghari G, Azizi F. Broccoli sprouts powder could improve serum triglyceride and oxidized LDL/LDL-cholesterol ratio in type 2 diabetic patients: A randomized double-blind placebocontrolled clinical trial. Diabetes Res Clin Pract 2012; 96: 348-54.

60. Bahadoran Z, Tohidi M, Nazeri P, Mehran M, Azizi F, Mirmiran P. Effect of broccoli sprouts on insulin resistance in type 2 diabetic patients: A randomized double-blind clinical trial. Int J Food Sci Nutr 2012; 63: 767-71.

61. Qin F, Siwik DA, Luptak I, et al. The polyphenols resveratrol and s17834 prevent the structural and functional sequelae of diet-induced metabolic heart disease in mice. Circulation 2012; 125: 1757-64, S1-6.

62. Wu H, Li GN, Xie J, et al. Resveratrol ameliorates myocardial fibrosis by inhibiting ROS/Erk/TGF- $\beta$ /periostin pathway in STZ-induced diabetic mice. BMC Cardiovasc Disord 2016; 16: 5.

63. Wahab A, Gao K, Jia C, et al. Significance of resveratrol in clinical management of chronic diseases. Molecules 2017; 22: E1329.

64. Xia N, Daiber A, Forstermann U, Li H. Antioxidant effects of resveratrol in the cardiovascular system. Br J Pharmacol 2017; 174: 1633-46.

65. Li C, Xu X, Tao Z, Wang XJ, Pan Y. Resveratrol dimers, nutritional components in grape wine, are selective ros scavengers and weak Nrf2 activators. Food Chem 2015; 173: 21823.

66. Ungvari Z, Bagi Z, Feher A, et al. Resveratrol confers endothelial protection via activation of the antioxidant transcription factor Nrf2. Am J Physiol Heart Circ Physiol 2010; 299: H18-24.

67. Delucchi F, Berni R, Frati C, et al. Resveratrol treatment reduces cardiac progenitor cell dysfunction and prevents morpho-functional ventricular remodeling in type-1 diabetic rats. PLoS One 2012; 7: e39836.

68. Mohammadshahi M, Haidari F, Soufi FG. Chronic resveratrol administration improves diabetic cardiomyopathy in part by reducing oxidative stress. Cardiol J 2014; 21: 39-46.

69. Beaudoin MS, Perry CG, Arkell AM, et al. Impairments in mitochondrial palmitoyl-coa respiratory kinetics that precede development of diabetic cardiomyopathy are prevented by resveratrol in ZDF rats. J Physiol 2014; 592: 2519-33.

70. Vella RK, Pullen C, Coulson FR, Fenning AS. Resveratrol prevents cardiovascular complications in the SHR/STZ rat by reductions in oxidative stress and inflammation. Biomed Res Int 2015; 2015: 918123.

71. Fang WJ, Wang CJ, He Y, Zhou YL, Peng XD, Liu SK. Resveratrol alleviates diabetic cardiomyopathy in rats by improving mitochondrial function through PGC- $1 \alpha$ deacetylation. Acta Pharmacol Sin 2018; 39: 59-73. 
72. Oyenihi OR, Oyenihi AB, Adeyanju AA, Oguntibeju OO. Antidiabetic effects of resveratrol: The way forward in its clinical utility. J Diabetes Res 2016; 2016: 9737483.

73. Christenson J, Whitby SJ, Mellor D, et al. The effects of resveratrol supplementation in overweight and obese humans: A systematic review of randomized trials. Metab Syndr Relat Disord 2016; 14: 323-33.

74. Na LX, Li Y, Pan HZ, et al. Curcuminoids exert glucoselowering effect in type 2 diabetes by decreasing serum free fatty acids: A double-blind, placebo-controlled trial. Mol Nutr Food Res 2013; 57: 1569-77.

75. Chuengsamarn S, Rattanamongkolgul S, Phonrat B, Tungtrongchitr R, Jirawatnotai S. Reduction of atherogenic risk in patients with type 2 diabetes by curcuminoid extract: A randomized controlled trial. J Nutr Biochem 2014; 25: 144-50.

76. Yang $\mathrm{H}, \mathrm{Xu} \mathrm{W}$, Zhou Z, et al. Curcumin attenuates urinary excretion of albumin in type ii diabetic patients with enhancing nuclear factor erythroid-derived 2-like 2 (Nrf2) system and repressing inflammatory signaling efficacies. Exp Clin Endocrinol Diabetes 2015; 123: 360-7.

77. Yu W, Wu J, Cai F, et al. Curcumin alleviates diabetic cardiomyopathy in experimental diabetic rats. PLoS One 2012; 7: e52013.

78. Soetikno V, Sari FR, Sukumaran V, et al. Curcumin prevents diabetic cardiomyopathy in streptozotocin-induced diabetic rats: Possible involvement of PKC-MAPK signaling pathway. Eur J Pharm Sci 2012; 47: 604-14.

79. Karuppagounder V, Arumugam S, Giridharan VV, et al. Tiny molecule, big power: Multi-target approach for curcumin in diabetic cardiomyopathy. Nutrition 2017; 34: 47-54.

80. Aziz MT, El Ibrashy IN, Mikhailidis DP, et al. Signaling mechanisms of a water soluble curcumin derivative in experimental type 1 diabetes with cardiomyopathy. Diabetol Metab Syndr 2013; 5: 13.

81. Linker RA, Lee DH, Ryan S, et al. Fumaric acid esters exert neuroprotective effects in neuroinflammation via activation of the Nrf2 antioxidant pathway. Brain 2011; 134: 678-92.

82. Sun H, Zhu J, Lin H, Gu K, Feng F. Recent progress in the development of small molecule Nrf2 modulators: A patent review (2012-2016). Expert Opin Ther Pat 2017; 27: 763-85.

83. Brennan MS, Patel H, Allaire N, et al. Pharmacodynamics of dimethyl fumarate are tissue specific and involve Nrf2dependent and -independent mechanisms. Antioxid Redox Signal 2016; 24: 1058-71.

84. Ganeshpurkar A, Saluja AK. The pharmacological potential of rutin. Saudi Pharm J 2017; 25: 149-64.

85. Wang YB, Ge ZM, Kang WQ, Lian ZX, Yao J, Zhou CY. Rutin alleviates diabetic cardiomyopathy in a rat model of type 2 diabetes. Exp Ther Med 2015; 9: 451-5.

86. Gao HC, Zhu K, Gao HM, et al. Role of tissue transglutaminase in the pathogenesis of diabetic cardiomyopathy and the intervention effect of rutin. Exp Ther Med 2015; 9: 11038.

87. Guimaraes JF, Muzio BP, Rosa CM, et al. Rutin administration attenuates myocardial dysfunction in diabetic rats. Cardiovasc Diabetol 2015; 14: 90.

88. Saklani R, Gupta SK, Mohanty IR, Kumar B, Srivastava S, Mathur R. Cardioprotective effects of rutin via alteration in TNF- $\alpha$, CRP, and BNP levels coupled with antioxidant effect in STZ-induced diabetic rats. Mol Cell Biochem 2016; 420: 65-72.

89. Huang R, Shi Z, Chen L, Zhang Y, Li J, An Y. Rutin alleviates diabetic cardiomyopathy and improves cardiac function in diabetic APOEknockout mice. Eur J Pharmacol 2017; 814: 151-60.

90. Yu Y, Zheng G. Troxerutin protects against diabetic cardiomyopathy through NFKb/Akt/IRS1 in a rat model of type 2 diabetes. Mol Med Rep 2017; 15: 3473-8.
91. Semwal DK, Semwal RB, Combrinck S, Viljoen A. Myricetin: A dietary molecule with diverse biological activities. Nutrients 2016; 8: 90

92. Liao HH, Zhu JX, Feng H, et al. Myricetin possesses potential protective effects on diabetic cardiomyopathy through inhibiting Iкb $\alpha / \mathrm{NF} \kappa b$ and enhancing Nrf2/HO-1. Oxid Med Cell Longev 2017; 2017: 8370593.

93. Jung HY, Lee D, Ryu HG, et al. Myricetin improves endurance capacity and mitochondrial density by activating SIRT1 and PGC-1 $\alpha$. Sci Rep 2017; 7: 6237.

94. Rushworth SA, Zaitseva L, Murray MY, Shah NM, Bowles KM, MacEwan DJ. The high Nrf2 expression in human acute myeloid leukemia is driven by NFKb and underlies its chemo-resistance. Blood 2012; 120: 5188-98.

95. Guo Y, Shen L. Overexpression of Nrf2 is correlated with prognoses of patients with malignancies: A meta-analysis. Thorac Cancer 2017; 8: 558-64.

96. DeNicola GM, Chen PH, Mullarky E, et al. Nrf2 regulates serine biosynthesis in non-small cell lung cancer. Nat Genet 2015; 47: 1475-81.

97. Satoh H, Moriguchi T, Saigusa D, et al. Nrf2 intensifies host defense systems to prevent lung carcinogenesis, but after tumor initiation accelerates malignant cell growth. Cancer Res 2016; 76: 3088-96.

98. Satoh H, Moriguchi T, Takai J, Ebina M, Yamamoto M. Nrf2 prevents initiation but accelerates progression through the KRAS signaling pathway during lung carcinogenesis. Cancer Res 2013; 73: 4158-68.

99. Castillo JJ, Mull N, Reagan JL, Nemr S, Mitri J. Increased incidence of non-Hodgkin lymphoma, leukemia, and myeloma in patients with diabetes mellitus type 2: A meta-analysis of observational studies. Blood 2012; 119: 4845-50.

100. Ohkuma T, Peters SAE, Woodward M. Sex differences in the association between diabetes and cancer: A systematic review and meta-analysis of 121 cohorts including 20 million individuals and one million events. Diabetologia 2018; 61: 214054.

101. Wang H, Liu X, Long M, et al. Nrf2 activation by antioxidant antidiabetic agents accelerates tumor metastasis. Sci Transl Med 2016; 8: 334ra51.

102. Cuadrado A, Martin-Moldes Z, Ye J, Lastres-Becker I. Transcription factors Nrf2 and NFKb are coordinated effectors of the RHO family, GTP-binding protein RAC1 during inflammation. J Biol Chem 2014; 289: 15244-58.

103. Steele ML, Fuller S, Patel M, Kersaitis C, Ooi L, Munch G. Effect of Nrf2 activators on release of glutathione, cysteinylglycine and homocysteine by human u373 astroglial cells. Redox Biol 2013; 1: 441-5.

104. Chin MP, Bakris GL, Block GA, et al. Bardoxolone methyl improves kidney function in patients with chronic kidney disease stage 4 and type 2 diabetes: Post-hoc analyses from bardoxolone methyl evaluation in patients with chronic kidney disease and type 2 diabetes study. Am J Nephrol 2018; 47: 40-7.

105. Stockwell BR, Friedmann Angeli JP, Bayir H, et al. Ferroptosis: A regulated cell death nexus linking metabolism, redox biology, and disease. Cell 2017; 171: 273-85.

106. Dixon SJ, Lemberg KM, Lamprecht MR, et al. Ferroptosis: An iron-dependent form of nonapoptotic cell death. Cell 2012; 149: 1060-72.

107. Fan Z, Wirth AK, Chen D, et al. Nrf2-keap1 pathway promotes cell proliferation and diminishes ferroptosis. Oncogenesis 2017; 6: e371.

108. Abdalkader M, Lampinen R, Kanninen KM, Malm TM, Liddell JR. Targeting Nrf2 to suppress ferroptosis and mitochondrial dysfunction in neurodegeneration. Front Neurosci 2018; 12: 466 